$\begin{array}{cl}\begin{array}{c}\text { Revue } \\ \text { de } / \text { histoire } \\ \text { des religions }\end{array} & \text { Revue de l'histoire des religions } \\ & \begin{array}{l}1 \mid 2014 \\ \text { Varia }\end{array}\end{array}$

\title{
La doctrine de la grâce de saint Augustin. Aspects pastoraux et spéculatifs
}

Augustine's Doctrine of Grace. Pastoral and Systematic-Theological Perspectives

\section{Anthony Dupont}

\section{(2) OpenEdition}

\section{Journals}

Édition électronique

URL : http://journals.openedition.org/rhr/8193

DOI : $10.4000 /$ rhr.8193

ISSN : 2105-2573

Éditeur

Armand Colin

\section{Édition imprimée}

Date de publication : 1 mars 2014

Pagination : 47-70

ISBN : 978-2200929107

ISSN : 0035-1423

\section{Référence électronique}

Anthony Dupont, "La doctrine de la grâce de saint Augustin. Aspects pastoraux et spéculatifs ", Revue de l'histoire des religions [En ligne], 1 | 2014, mis en ligne le 01 mars 2017, consulté le 19 avril 2019.

URL : http://journals.openedition.org/rhr/8193 ; DOI : 10.4000/rhr.8193 


\section{La doctrine de la grâce de saint Augustin Aspects pastoraux et spéculatifs}

Le présent article étudie l'exégèse augustinienne de Jean 1,12 et 6,44 comme un double cas-test, consacré au thème de son enseignement sur la foi comme grâce, afin d'évaluer les différences/similitudes de l'œuvre d'Augustin entre les différentes périodes (en particulier avant et pendant la controverse pélagienne) et entre les genres (en particulier les sermons et les écrits systématiques-polémiques).

Augustine's Doctrine of Grace.

Pastoral and Systematic-Theological Perspectives

The present article studies Augustine's exegesis of John 1,12 and 6,44 as a double test case to evaluate the differences/similarities between the different periods (especially before and during the Pelagian controversy) and the genres (esp. sermons and systematic-polemical writings) of Augustine's oeuvre concerning his teachings on faith as grace. 
Existe-t-il une différence entre le traitement de la grâce dans les sermons d'Augustin (sermones ad populum, enarrationes et tractatus - c'est-à-dire dans ses initiatives pastorales destinées à exhorter sa communauté à mener une vie active éthique, fidèle et pieuse) et dans ses écrits spéculatifs et systématiques - particulièrement ses écrits anti-pélagiens, dans lesquels Augustin a souligné la nécessité absolue et la nature entièrement inclusive, gratuite et omniprésente de la grâce dans tous les aspects de la vie chrétienne? Pierre-Marie Hombert a montré que, avant même que la controverse pélagienne mît en question la relation entre la grâce divine et le libre arbitre humain, la conception augustinienne d'une grâce totalement gratuite (car l'humanité mérite seulement la punition, alors que tout le bien, en l'homme, est reçu de Dieu) n'était pas absente de ses discours homilétiques. Cependant, des thèmes plus spécifiques (comme les questions de la prédestination, ou de l'initium fidei conçu comme un effet produit par la grâce sur la uoluntas humaine) n'apparaissaient pas dans ces premiers sermons, alors qu'Augustin les avait déjà exposés dans ses écrits spéculatifs/systématiques de cette période (particulièrement dans l'Ad Simplicianum de 396/398)1. Donato Ogliari admet que la théologie spéculative des traités anti-pélagiens de saint Augustin et la théologie pratique de ses sermons (à l'époque de la controverse pélagienne) sont situées à différents niveaux, mais il note que, plutôt qu'une distinction nette entre ces deux domaines, il existe une «interaction féconde». Il observe que tous les sujets (même les plus sensibles, comme par exemple le problème de la damnation des bébés morts avant d'avoir été baptisés ou la prédestination entendue comme l'élection d'un nombre fixe de fidèles) étudiés dans les traités anti-pélagiens sont également discutés à cette période dans ses sermons².

À première vue, il ne semble pas opportun, sur un plan pastoral, d'inviter la communauté des fidèles à faire de leur mieux pour

1. Pierre-Marie Hombert, «Augustin, prédicateur de la grâce au début de son épiscopat», Augustin Prédicateur (395-411). Actes du Colloque International de Chantilly, 5-7 septembre, 1996 (éd. Goulven Madec) (Paris, 1996), 217-245.

2. Donato Ogliari, Gratia et Certamen. The Relationship between Grace and Free Will in the Discussion of Augustine with the so-called Semipelagians, [BETL, 169], (Leuven, 2003), spéc. 329-330, n. 137. 
mener une vie juste et, simultanément, de leur expliquer dans ses sermons que tous les efforts humains ne sont couronnés de succès que si la grâce de Dieu leur vient en aide. On admet généralement qu'Augustin ne s'est pas privé d'évoquer des sujets théologiques complexes dans ses sermons; néanmoins, on doit se demander si cela vaut également pour la question de la grâce ${ }^{3}$. Par ailleurs, une partie des sermons d'Augustin présente (plus ou moins explicitement) un objectif et un contenu polémiques, et ont pour but de lutter contre les thèses des manichéens, des donatistes, des pélagiens, entre autres ${ }^{4}$. Dans ces conditions, qu'en est-il de la doctrine anti-pélagienne de la grâce: Augustin la développe-t-elle, jusqu'en ses conséquences les plus radicales, dans ses sermons?

Pour répondre à cette question, cet article examinera la présence de deux versets bibliques, Jean 1,12 ( «Dedit illis potestatem filios Dei fieri, credentibus in eum») et Jean 6,44 («Nemo uenit ad me, nisi Pater, qui misit me, traxerit eum») et le traitement qui leur est réservé dans l'œuvre d'Augustin. La théologie d'Augustin était en effet substantiellement biblique: l'Écriture constituait le cœur de tous ses écrits, qu'ils soient spéculatifs, pastoraux, ou polémiques. L'étude de l'utilisation par Augustin de versets bibliques spécifiques dans l'ensemble de son opus offre en conséquence la possibilité de comparer les différents genres, puisque la Bible représente le facteur constant dans tous ses écrits. Une étude attentive de ces deux versets nous permettra de détecter les similarités et les différences entre les écrits antérieurs et postérieurs à 411/412, ainsi que ce qui sépare les différents genres de textes composés par Augustin (spécialement les sermons et les traités anti-pélagiens) à propos d'un aspect spécifique de la doctrine augustinienne de la grâce: le statut de la foi.

Une des questions cruciales dans le débat pélagien concerne en effet la nature de la foi : le choix de la foi juste et la persévérance dans

3. Christine Mohrmann, «Saint Augustin prédicateur», Études sur le Latin des Chrétiens, 1 (éd. Christine Mohrmann) (Rome, 1958), 391-402. Alexandre Olivar, La Predicación Cristiana Antigua, [Biblioteca Herder. Sección de Teología y Filosofía, 189], (Barcelona, 1991), 383-385. Michele Pellegrino, «General Introduction», Sermons I (1-19). On the Old Testament (ed. John E. Rotelle), [WSA, 3/1], (New York, 1990), 13-163, 56-73. Henri Rondet, «La théologie de saint Augustin prédicateur», BLE 72 (1971), 81-105, 241-257.

4. Par exemple, Gert Partoens discute le $s .163$ comme un exemple de polémique anti-pélagienne. Gert Partoens, «Sermones», Augustinus Handbuch (ed. Volker Henning Drecoll) (Tübingen, 2007), 393-416, 410-416. 
cette voie relèvent-ils de l'entière responsabilité de l'humanité? La foi et la persévérance sont-elles des dons de Dieu indépendants de la volonté humaine, ou le produit conjoint de l'initiative divine et d'une réponse humaine? Les versets bibliques cités semblent répondre à cette question en soulignant la prééminence de Dieu dans l'acte de foi. À ce titre, ils nous mènent au centre de la pensée d'Augustin sur la fides et la gratia. L'étude de l'exégèse augustinienne de Jean 1,12 et 6,44 permettra donc d'évaluer, sur la question du rapport entre foi humaine et grâce divine, les différences et les points communs entre les différentes périodes et entre les différents genres représentés dans l'œuvre d'Augustin.

\section{JEAN 1,12}

\section{Les écrits antérieurs à 411/412}

Augustin utilise Jean 1,12 dans des contextes variés. Dans la majorité de ses commentaires sur le verset, son explication s'attache à éclairer le thème de la gratia et le rapport entre gratia et fides. Dans ses premiers écrits cependant, le verset permet d'illustrer un certain nombre d'antithèses: les amis de ce monde uersus les fils de Dieu ${ }^{5}$, les Juifs (qui ont démontré par leurs péchés qu'ils ne veulent pas être les enfants de Dieu) uersus les chrétiens (qui sont, par contraste, les enfants de Dieu) $)^{6}$.

Dans d'autres textes, Augustin se montre plus précis, expliquant Jean 1,12 dans le sens d'une potestas filios Dei fieri accordée comme un don à l'humanité. Avant la controverse pélagienne, il a déjà observé dans le Contra Adimantum (394) que Dieu a accordé aux êtres humains la potestas de devenir les enfants de Dieu - autrement dit que Dieu renouvelle les personnes humaines selon l'imago Dei en laquelle ils ont été créés ${ }^{7}$. La juxtaposition des pôles actifs et passifs de la foi sur la base de Jean 1,12, avec l'accent mis sur le rôle humain, figure en première instance dans les écrits les

5. Augustin, uera rel. 65 (390/391). Les dates des écrits d'Augustin sont celles collectées dans: Allan D. Fitzgerald (ed.), Augustine through the Ages. An Encyclopedia (Grand Rapids MI/Cambridge, 1999).

6. Augustin, s. dom. in m. 2.15 (393/395).

7. Augustin, c. Adim. 5. 
plus anciens d'Augustin. Dans son De sermone Domini in monte (393/395), par exemple, Augustin clarifie le statut de la fides en faisant référence à ce verset. Il souligne que la foi est une tâche humaine: si les hommes accomplissent ce que Dieu leur demande, ils sont adoptés comme les enfants de Dieu. Le Dieu qui nous a amenés à l'existence fait désormais de nous ses enfants adoptifs, afin que la personne humaine puisse profiter de la vie éternelle avec lui. «Itaque non ait: facite ista, quia estis filii, sed: facite ista, ut sitis filii » ${ }^{8}$. Le Contra Secundinum Manicheum (399) stipule clairement que la personne humaine est adoptée comme un enfant de Dieu, en vertu de sa grâce miséricordieuse. L'homme a reçu cette grâce de Dieu en croyant dans le Fils. Augustin ajoute, en outre, que le contenu précis de cette foi dépend du travail individuel de chaque individu?.

\section{Corpus homilétique}

\section{Les sermons antérieurs à la controverse pélagienne, ou sans lien avec elle}

Dans le Sermo 342 (non daté) ${ }^{10}$, nous trouvons un exemple supplémentaire d'antithèse (cf. supra), à savoir celle entre les Juifs arrogants (la branche coupée - par leur refus d'accepter le Christ, ils sont devenus des étrangers) et les gentes humbles (l'olivier sauvage greffé avec le centurion, par exemple) ${ }^{11}$.

Il est ainsi fréquemment fait allusion à Jean 1,12 dans le contexte de la gratia, et en particulier dans des sermons antérieurs à la controverse pélagienne, dans lesquels ce verset est utilisé pour

8. Augustin, s. dom. in m. 1.78. [CChrSL 35:88/1919-1920].

9. Augustin, c. Sec. 5. Cf. Augustin, ep. 140.11 (411/412). La foi dans le Christ apporte la renaissance par la grâce spirituelle.

10. Rebillard:-, Gryson:-, Hombert: -. Bien qu'il y ait beaucoup de littérature sur la datation des Sermones ad populum spécifiques, je renvoie pour chaque datation d'un sermo à trois aperçus chronologiques récents, soit R. Gryson, P.-M. Hombert, et É. Rebillard. Roger Gryson - Bonifatius Fischer - Hermann Josef Frede, Répertoire général des auteurs ecclésiastiques Latins de l'Antiquité et du Haut moyen âge, [VL 1/1], (Freiburg, $\left.{ }^{5} 2007\right)$. Pierre-Marie Hombert, Nouvelles recherches de chronologie augustinienne, [CEA Série Antiquité 163], (Paris, 2000). Éric Rebillard, «Sermones», Augustine through the Ages. An Encyclopedia (ed. Allan D. Fitzgerald) (Grand Rapids MI/Cambridge, 1999), 773-792.

11. Cf. l'antithèse entre ceux qui acceptent le Christ et ceux qui ne l'acceptent pas: Augustin, Io. eu. tr. 3.6 (406/407). 
souligner le fait que Dieu adopte gracieusement la communauté des fidèles pour en faire ses fils. Augustin déclare que les personnes humaines deviennent les enfants de Dieu par la grâce de l'adoption - en contraste avec le Christ, qui est Fils de Dieu par nature. Il ne propose toutefois pas de développement systématique sur le rôle de la foi dans ce processus d'adoption ${ }^{12}$. Dans le Sermo 121.3-4 $(404-410)^{13}$, il souligne la distinction entre les gentes et les Juifs en faisant valoir que seuls les premiers ont reçu la grâce à cause de leur foi. Néanmoins, il ne précise pas si cette foi est une grâce ou un mérite ${ }^{14}$.

Dans le corpus homilétique d'Augustin, nous trouvons des traitements différents de ce verset. L'accent se trouve particulièrement mis sur la part de l'iniative humaine dans la foi dans trois Sermones ad populum. Le Sermo 360B (403) ${ }^{15}$ souligne la responsabilité humaine dans la foi et insiste sur le fait que c'est de cette responsabilité que relève notre guérison par le médecin, afin de devenir un enfant de Dieu - avec une allusion à Jean 1,12 - et de ne plus se contenter de rester simplement «humain ${ }^{16}$. Le traitement du medicus (le Christ) est administré et, dans ce sens, il relève d'une initiative divine. Cependant, la personne humaine doit accepter de se soumettre à un traitement douloureux et de

12. Augustin, Io. eu. tr. 2.13 (406/407). La foi est comme un héritage qui n'est pas réduit par le nombre de bénéficiaires. Augustin, en. Ps. 49.2 (Müller: -, Zarb: carême 412, Rondet: 412, La Bonnardière: 400-411, Hombert: fin 412). Dans le contexte de la justification et de la déification, Augustin argumente, sur base de Jean 1,12, que les fidèles deviennent les enfants de Dieu. Ceci se passe par l'intermédiaire de l'adoption, par le don gratuit de Dieu, et non par nature (à l'exception du Christ). Il souligne également ici qu'il s'agit d'un héritage qui n'est pas réduit par le nombre (de plus en plus grand) des bénéficiaires et il établit un lien avec la différence entre la confessio de Pierre (Matthieu 16,16) et celle des démons (Marc 1,24, Luc 4,41). Les dates des Enarrationes sont celles proposées dans: Hildegund Müller, «Enarrationes in Psalmos, A. Philologische Aspekte», Aug-Lex II, 5/6 (2001), 804-838. Voir aussi : Augustin, c. Faust. 3.3 (397/399); s. Dolbeau 22.18 (s. 341 Aug., Rebillard: -, Gryson: 12/12/403, Hombert: -); trin. 5.17 (Hombert: 416); 13.12 (Hombert: 420-426/427).

13. Rebillard: avant 410, Gryson : circa 404 ?, Hombert :-

14. Augustin, s. 121.3. «Numquam aduertistis quemadmodum canes lapides unctos lingunt? Sic sunt omnes simulacrorum cultores. Sed uenit uobis gratia. Quotquot autem receperunt eum, dedit eis potestatem filios Dei fieri [Jean 1,12]. Ecce habetis modo natos: dedit eis potestatem filios Dei fieri [Jean 1,12]. Quibus dedit? His qui credunt in nomine eius [Jean 1,12]». [SC 116:228/66-71].

15. Augustin, $s$. Dolbeau 25, Rebillard: -, Gryson: à Rome, après la visite d'Honorius, au début de Décembre 403, Hombert: -.

16. Augustin, s. 360B.15. 
persévérer dans les efforts qu'il exige d'elle ${ }^{17}$. En d'autres termes, comme la foi se rapporte à ce que la personne humaine ne peut pas voir ${ }^{18}$, Augustin conseille: «Croyez que vous pourriez être capable de voir, si bien que vous puissiez mériter cette capacité de voir». La vue est donc méritée par la foi, comme une récompense qui vient couronner un travail ${ }^{19}$. Le Sermo 166 (postérieur à 410) ${ }^{20}$ cite également Jean 1,12, mais sans établir de lien avec le fait de croire. Augustin demande à ses auditeurs de ne pas mentir et de ne pas se contenter d'être un homme comme l'a été Adam: au contraire, il les exhorte à dire la vérité comme le Christ. Dieu, après tout, désire que nous devenions ses enfants - non par nature, mais par adoption ${ }^{21}$. À ce titre, Jean 1,12 fait ici allusion à la responsabilité humaine, sans l'identifier spécifiquement à la foi. Le Sermo 97A (non daté) ${ }^{22}$ cite Jean 1,12, mais seulement en partie: «dedit eis potestatem filios Dei fieri», dans un appel adressé aux catéchumènes pour les exhorter au baptême, afin qu'ils viennent à la grâce, et reçoivent la potestas dont il est question dans le verset johannique. L'actif (ueni) et le passif (accepisti) sont ici mêlés ${ }^{23}$. Au début de son homélie, par exemple, Augustin déclare que le Christ, le medicus, transforme l'humanité impie en une humanité pieuse (cf. Rom. 4,5) ${ }^{24}$. Toutefois, une telle foi est une tâche que l'homme doit accomplir. Il est en effet nécessaire de croire en quelqu' un que l'on ne peut voir, afin qu'en croyant nous puissions mériter de voir ce que nous croyons. Cela est exprimé avec

17. Augustin, s. 360B.16. Le caractère de grâce se manifeste aussi à l'évidence dans l'hypothèse où l'œil intérieur (le cœur) doit être donné à la lumière intérieure pour pouvoir voir (Augustin, s. 360B.10; 15) et que le medicus (le Christ) purifie le cour avec/par la foi (Actes 15,9).

18. Augustin, s. 360B.20. Cette foi est facilitée dans une certaine mesure par le fait que (beaucoup des) les anciennes promesses ont déjà été réalisées et que seules quelques-unes demeurent. Dans le passé, pour ceux qui n'ont jamais vu ce que les fidèles voient maintenant (par exemple, l'œuvre du Christ à la conversion des gentes), la foi était quelque chose de grand et de louable. Maintenant que la foi n'est plus si digne de louange, refuser de croire aujourd'hui en est devenu d'autant plus condamnable. Augustin insiste dans ce paragraphe sur le fait que la personne humaine doit croire d'abord et seulement ensuite voir.

19. Augustin, s. 360B.20.

20. Rebillard: après 410, Gryson: le dimanche après la Pentecôte, après 410, Hombert : -

21. Augustin, s. 166.4 .

22. Augustin, s. Casin. 2.114-115, Rebillard: -, Gryson: -, Hombert: -.

23. Augustin, s. 97A.4.

24. Augustin, s. 97A.1. 
concision: «Meritum uisionis, fides est; merces fidei, uisio est $\gg^{25}$. Augustin veut signifier ici que seule la foi dans le Dieu invisible peut avoir pour résultat la vision de Dieu après la Résurrection. Dans notre analyse de la première utilisation de ce verset, nous avons été en mesure d'observer qu' Augustin l'utilise pour indiquer que l'homme doit s'assurer de l'orthodoxie de sa foi, afin que Dieu puisse l'adopter et le transformer: c'est cet usage du verset qui est évidemment majoritaire dans les sermones.

Quant à l' affirmation selon laquelle le fait de croire, et l'orthodoxie de cette foi, relèvent également d'un don de la grâce, elle se trouve rarement dans le contexte de Jean 1,12 hors de la période de la controverse pélagienne. De fait, nous ne la rencontrons explicitement qu'à une seule occasion, dans l'Enarratio in Psalmum 120 (405$411)^{26}$. Le fait d'être assis à la droite de Dieu (Ps. 120,5), signifie être «fils et fille» de Dieu: l'homme a reçu cette potestas de Dieu comme un don - et ici Augustin cite Jean 1,12. Augustin conclut donc que l'homme a reçu la foi de Dieu: outre cette potestas, le croyant reçoit également la persévérance dans cette foi. La potestas de la main droite - la foi - est donc non seulement reçue, mais préservée par Dieu ${ }^{27}$. De façon moins explicite, Augustin étudie dans l'Enarratio sur le Psaume 72 (13-14/09/avant 412) ${ }^{28}$, le verset 24, dans lequel le psalmiste déclare que Dieu l'a pris par la main droite. Selon le prédicateur, la main droite représente la potestas d'être avec Dieu, de devenir filius Dei. Cela ne se produit pas en fonction de ses propres merita, cependant, mais selon la uoluntas de

25. Augustin, s. 97A.2. Augustin, s. 97A.3 se réfère aux dimensions actives et passives pour éviter le péché. Nous n'avons plus aucun pouvoir sur nos péchés passés. Ceux-ci sont pardonnés par le baptême. Mais les péchés futurs peuvent être activement évités. Ceux qui pensent qu'ils sont incapables de le faire doivent chercher refuge dans le Christ. Le paragraphe suivant (Augustin, s. 97A.4) stipule en des termes similaires que les péchés passés sont pardonnés par le baptême et que la personne humaine est censée effectuer de bonnes œuvres après le baptême (c'est-à-dire, après la foi).

26. Müller: pas avant 405 et pas plus tard que juin 411 , Zarb: décembre 412 , Rondet: printemps 408 (ou 407)/415, Le Landais: décembre 414-août 415, La Bonnardière: entre l'hiver 406 et la Pâques 407 ?, Poque: février/mars 407.

27. Augustin, en. Ps. 120.11. La main droite fait allusion à Job 1,11. Les impii sont assis sur la main gauche. Augustin ajoute que les chrétiens sont des gens qui sont venus à la foi et qui marchent dans la foi, bien qu'ils sont faibles et soumis à la tentation.

28. Müller : 13-14/09/avant 412, Zarb : 411, Rondet : 411/412, La Bonnardière : 410 
Dieu ( 1 Tim. 1,13 et 1 Cor. 15,10$)^{29}$. Il va sans dire que ce thème ne disparaît pas avec le début de la controverse pélagienne.

\section{Les sermons contemporains de la controverse pélagienne}

Le Sermo 143 (410-412) $)^{30}$ se situe au début de la controverse pélagienne, et tend à mettre l'accent sur la foi comme grâce. Ce sermon traite de la péricope Jean 16,7-11: la promesse du Christ d'envoyer le Saint-Esprit pour porter un jugement sur le monde ${ }^{31}$. Augustin remarque que tous les péchés sont remis lorsque l'on croit dans le Seigneur. Il se demande alors sur quel péché le Saint-Esprit portera un jugement à son avènement, en gardant à l'esprit que le Christ avait annoncé en Jean 16,8: «arguet mundum de peccato, et de iustitia, et de iudicio?». C'est Jean 16,9 qui lui fournit la réponse: il concerne le péché de l'incroyance en Jésus-Christ. C'est le péché fondamental, celui qui relie tous les péchés entre eux. Ce n'est que dans la mesure où les fidèles conservent leur foi dans le Christ - par lequel ils deviennent les enfants de Dieu, et les cohéritiers de Christ par l'adoption (en ligne avec Jean 1,12: «dedit illis potestatem filios Dei fieri, credentibus in eum») - qu'ils ne pèchent pas. Le péché de l'incroyance a été ajouté aux péchés de ceux qui ont refusé de croire après l'avènement du Christ (cf. Jean 15,22 ), et ce péché d'incroyance relie ensemble tous leurs autres péchés. Selon Augustin, le meilleur exemple en est l'incroyance des Juifs $^{32}$. Pour ceux qui croient, l'absence de l'incroyance est la raison pour laquelle leurs péchés ont été pardonnés. Augustin déclare donc que le salut dépend de la présence ou de l'absence de la foi en Jésus-

29. Augustin, en. Ps. 72.30. En faisant allusion à la main dans Job 1,11, Augustin argue dans son commentaire sur Ps. 72,24 de ce que le psalmiste symbolise les chrétiens juifs, les chrétiens qui, comme les apôtres, sont venus parmi le peuple juif. La main droite contraste avec la gauche, cette dernière représentant la concupiscentia terrestre.

30. Rebillard: 410-412, Gryson: 410/412.

31. «Ego ueritatem dico uobis, expedit uobis ut ego uadam. Si enim ego non abiero, Paracletus non ueniet ad uos: si autem abiero, mittam eum ad uos. Et cum uenerit ille, arguet mundum de peccato, et de iustitia, et de iudicio. De peccato quidem, quia non crediderunt in me: de iustitia uero, quia ad Patrem uado, et iam non uidebitis me: de iudicio autem, quia princeps huius mundi iudicatus est». [Jean 16,7-11 en Augustin, s. 143.1, PL 38:85/12-20].

32. Anthony Dupont, «The Relation between Pagani, Gentes and Infideles in Augustine's Sermones ad Populum: A Case Study of Augustine's Doctrine of Grace», $\operatorname{Aug}(L)$ 58/1-2 (2008), 95-126. 
Christ $^{33}$. Le Saint-Esprit vient et jugera selon le péché d'incroyance. L'arrivée du Saint-Esprit est un don de la grâce, conférée aux fidèles. Mais dans quelle mesure exacte nous devrions considérer la foi elle-même comme une grâce, comme un don du Saint-Esprit, voilà qui n'est pas encore tout à fait clair. Nous pourrions même soutenir qu'Augustin accentue la confusion en affirmant: "Non autem esset meritum grande credentium et beatitudo gloriosa, si semper Dominus in resuscitato corpore humanis oculis appareret $\gg^{34}$. Est-ce donc un mérite humain de croire dans le Seigneur sans avoir vu son corps ressuscité? Dans le prolongement de ce sermo, par ailleurs, Augustin ne déclare pas clairement que la foi elle-même est déjà un don de la grâce, bien qu'une telle position puisse être considérée comme la conséquence implicite du raisonnement qui sous-tend l'ensemble du sermon. Augustin continue son sermon en expliquant que le don (hoc magnum munus) du Saint-Esprit consiste en un désir spirituel pour le Christ - pas avec les yeux de la chair comme l'apôtre Thomas (cf. Jean 20,28-29), mais avec des esprits purifiés - sous la forme en laquelle il est l'égal du Père (et pas, par conséquent, sous sa forme incarnée). En collaboration avec Rom. 10,6-10, Augustin soutient que le salut consiste à croire

33. Augustin, s. 143.2. Depuis le début de la controverse pélagienne, Augustin argue de ce que l'impeccantia est possible en théorie, bien qu'aucun croyant ne puisse prétendre seul à cette réalité. Cette position se trouve au plus tôt dans pecc. mer., et c'est un point sur lequel il revient sans cesse, comme il ressort clairement, par exemple, de sa combinaison répétée de Matt. 6,12 et 1 Jean 1,8 durant cette période. La majorité des citations de Prov. 20,8-9 sont situées aussi dans ce contexte: "Cum enim rex iustus sederit in throno, quis gloriabitur castum se habere cor? Aut quis gloriabitur mundum se esse a peccato?». Ce verset ne se trouve pas dans le $s$. 143,2, bien que Pierre-Marie Hombert est enclin à argumenter que celui-ci contient l'idée que les gens ne devraient pas se glorifier dans leur propre iustitia ou prétendre qu'ils sont sans péché. Ils devraient se glorifier plutôt dans la misericordia du Seigneur. Sur la "glorification céleste en Dieu» dans Augustin, et plus précisément sur «se glorifier dans l'ultime miséricorde de Dieu », Hombert indique que le s. 143.2 (ensemble avec perf. iust 15.33) a la signification suivante: "L'heure du jugement sera celle de l'ultime décision: se glorifier en Dieu ou en soi-même; se glorifier de sa propre justice, en s'affirmant sans péché, ou «se glorifier dans la miséricorde» [...] ». (Voir le parallèle en Augustin, ep. 140.79 et perf. iust. 15.33.) Pierre-Marie Hombert, Gloria gratiae. Se glorifier en Dieu, principe et fin de la théologie augustinienne de la grâce, [CEA Série Antiquité 148], (Paris, 1996), 553 (n. 200), 554-555 (n. 203, 204, 206). Cf. AnneMarie La Bonnardière, Le Livre des Proverbes, [Biblia augustiniana: A.T. - CEA Série Antiquité 67], (Paris, 1973), 25-27, 70-74.

34. Augustin, s. 143.3. [PL 38:786/4]. 
dans le Christ ressuscité et dans la iustitia ${ }^{35}$. Quand il déclare alors que voir le Christ spiritaliter est un don de l'Esprit, nous pouvons conclure qu'il signifie que la foi en Jésus-Christ est une gratia ou un don de l'Esprit. Cela se confirme dans la suite du sermon: «Cum ergo hanc beatitudinem, qua non uidemus et credimus, nullo modo haberemus nisi eam a Spiritu Sancto acceperimus; $[\ldots] »^{36}$. En Sermo 306 (415-420), Augustin prêche que la décision de vivre une bonne vie (telle que l'on n'a plus besoin de mourir dans la peur - à l'image des martyrs) est une potestas accordée par Dieu à ceux qui croient. Jean 1,12 traite de l'octroi de cette potestas aux fidèles. Augustin suggère que cette foi est la iusta causa de la souffrance des martyrs ${ }^{37}$. L'homélie en question - dans le cadre général du thème de l'efficacité de la grâce sans les mérites précédents - déclare en bref que la potestas de vivre une bonne vie nous est donnée, mais elle n'explique pas comment cela est lié au fait de croire. Est-ce une potestas accordée sur la base d'une foi méritoire et antérieure, ou bien la foi est-elle conçue comme partie intégrante de la potestas ainsi donnée? Augustin n'est pas très clair sur ce point.

Avec encore plus de clarté et d'emphase, Augustin insiste, dans le Tractatus 53 sur l'Évangile selon saint Jean (circa 414), sur le fait que la foi elle-même est un don de Dieu. Le Christ a en effet dit: «Rogaui pro te, Petre, ne deficiat fides tua [Luc 22,32]». Selon Augustin, Il l'a fait pour que la personne humaine ne pense pas que la foi en tant que telle est une capacité du libre arbitre et qu'elle n'a pas besoin de l'aide de Dieu. Dans l'opinion d'Augustin, le sens de Jean 1,12 est similaire. Le verset indique clairement que la foi n'est pas dans notre propre potestas. Jean 1,12 est destiné à encourager les gens à reconnaître les beneficia qu'ils ont reçus de Dieu. Les gens devraient remercier Dieu à cause du don de la potestas et prier en même temps pour qu'ils ne retombent pas dans l'infirmitas. Augustin établit ici un lien avec Gal. 5,6. La foi, après tout, opère par l'amour, et cet amour nous est donné par Dieu ${ }^{38}$.

35. Augustin, s. 143.3. Augustin, s. 143.3: Paul a prêché le Saint-Esprit dans une ivresse sacrée (dans la lignée des prophètes et de tous les prédicateurs) et dépend donc de Dieu pour prêcher.

36. Augustin, s. 143.4. [PL 38:786/37-39].

37. Augustin, s. 306.2 (Rebillard: martyrs de Massa Candida 18/08/397, Gryson: martyrs de Massa Candida 18/08/415-420, Hombert: 415-420).

38. Augustin, Io. eu. tr. 53.8 (414). 


\section{Les écrits anti-pélagiens, postérieurs à 412}

Dans les Retractationes (426/427), Augustin propose une description nuancée de la relation entre les dimensions de la fides humaine et divine, dans le contexte de son analyse du Contra Adimantum. La personne humaine a la potestas de modifier sa propre uoluntas, pour la diriger vers Dieu. Cette potestas est un don de Dieu, et c'est grâce à elle que l'homme peut désirer ce qu'il désire; cette uoluntas est préparée par le Seigneur (Prov. 8,35). C'est dans ce sens que la potestas de la personne humaine nous est accordée par Dieu ${ }^{39}$.

Dès les débuts de la controverse pélagienne, il est fait allusion au verset en question dans le contexte de la gratia, bien que le lien entre la fides et la gratia ne soit pas toujours établi. Traitant du péché de l'homme, Augustin affirme dans l'Epistula 153 (413/414) que la personne humaine est mauvaise dans la mesure où elle est pécheresse et bonne dans la mesure où elle est un enfant de Dieu ${ }^{40}$. Une telle bonté, cependant, n'est pas quelque chose que nous possédons par nature, mais elle vient plutôt de Dieu, qui est bon en lui-même. La bonté de la personne humaine consiste à choisir avec sa propre volonté d'accepter la bonté de Dieu. A contrario, le refus de la bonté divine est la méchanceté humaine ${ }^{41}$. Les gens ne naissent pas comme enfants de Dieu par leur propre nature, mais deviennent enfants de Dieu par la grâce. Ce sont des enfants de Dieu dans la mesure où cette potestas leur a été accordée par Dieu parce qu'ils L'ont accepté et fait, pour ainsi dire, le choix de Le recevoir. Augustin a donc établi un équilibre entre l'initiative divine et la réponse de la libre volonté humaine. Mais ledit équilibre n'est, à ce stade, pas appliqué à la fides. L'Epistula 147 (413) indique clairement que la personne humaine ne voit Dieu en tant

39. Augustin, retr. 1.22.4.

40. Augustin, ep. 153.13. «Idcirco ipse Dominus quos dicit bonos propter participationem gratiae diuinae, eosdem etiam malos dicit propter uitia infirmitatis humanae, donec totum, quo constamus, ab omni uitiositate sanatum transeat in eam uitam, ubi nihil omnino peccabitur. Nam utique bonos docebat orare, non malos, quibus praecepit, ut dicerent: Pater noster, qui es in caelis [Matt. 6,9]; ex hoc enim boni, quo filii Dei non natura geniti sed gratia facti tamquam hi, quibus recipientibus eum dedit potestatem filios Dei fieri [Jean 1,12]». [CSEL 44:409/21410/8]. Avant ceci, Augustin a écrit dans l'ep. 153,12 que la personne humaine n'est pas sans péché dans cette vie.

41. Augustin, ep. 153.12. 
que Dieu que dans l'au-delà - et non pas pendant la vie terrestre. Cette potestas n'est pas gagnée. Au contraire, voir Dieu en tant que Dieu est une grâce ${ }^{42}$.

Dans la littérature spécifiquement anti-pélagienne, Augustin n'a pas tendance à employer directement Jean 1,12 pour décrire la fides comme un don de la grâce. Dans le De natura et gratia (carême 415), Augustin fait usage de ce verset pour faire allusion à la nature pécheresse de la personne humaine, en faisant valoir que nous ne pouvons certainement pas être des enfants de Dieu par nature, car la nature humaine est corrompue par le péché. Pour cette raison, la personne humaine peut seulement devenir un enfant de Dieu par la grâce ${ }^{43}$. Augustin poursuit en argumentant dans le De spiritu et littera ad Marcellinum (412) que la foi est un don du Saint-Esprit. Par la foi en Christ, les croyants sont libres, et agissent comme des enfants par amour. Ils ne sont donc plus des esclaves, comme ils l'étaient autrefois sous la loi. C'est la foi qui justifie le pécheur. Il s'agit d'une foi humble qui aspire à la justice, qui agit par amour et non par peur. La source de cet amour est le Saint-Esprit ${ }^{44}$. Jean 1,12 est cité dans le Contra duas epistulas Pelagianorum (421) en réaction contre les pelagiani, noui heretici, inimici gratiae: Augustin traite en premier lieu de leur interprétation de la relation entre la grâce et le libre arbitre. La potestas de vivre une bonne vie, selon Augustin, ne doit pas être attribuée à notre libre choix. Elle est offerte par la grâce de Dieu à travers le Christ, comme on peut le lire dans Jean $1,12^{45}$. Le reste du traité est encore plus explicite en la matière. Il est faux, à son avis, de soutenir que les personnes humaines sont capables de recevoir le Christ sur base de leur propre liberté de choix et sans l'aide de la grâce. Cela impliquerait que leur choix leur mériterait la potestas de devenir enfants de Dieu. Selon Augustin, les «pélagiens» déclarent faussement que la grâce est accordée en fonction du mérite. En d'autres termes, ils interprètent Jean 1,12 dans le sens que la foi mérite la grâce. Augustin introduit Jean 6,44 et Jean 6,64-65 à ce stade, les citations fonctionnant comme des contre-arguments et la preuve que la foi est un don. «Ille quippe trahitur ad Christum, cui datur ut credat in Christum. Datur

42. Augustin, ep. 147.23.

43. Augustin, nat. et gr. 77.

44. Augustin, spir. et litt. 56.

45. Augustin, c. ep. Pel. 1.5. 
ergo potestas, ut filii Dei fiant qui credunt in eum, cum hoc ipsum datur, ut credant in eum. Quae potestas nisi detur a Deo, nulla esse potest ex libero arbitrio, quia nec liberum in bono erit, quod liberator non liberauerit, sed in malo liberum habet arbitrium, cui delectationem malitiae uel occultus uel manifestus deceptor inseuit uel sibi ipse persuasit». En bref, la foi ne dépend pas du choix de l'homme, elle est plutôt un don de Dieu ${ }^{46}$.

Julien d'Éclane renvoie à l'usage par Augustin de Jean 1,12 dans le Contra duas epistulas Pelagianorum 1,5 (cf. supra), une référence qu'Augustin mentionne lui-même dans le Contra Iulianum opus imperfectum (429/430). Selon Augustin, la potestas de devenir un enfant de Dieu - de croire - est reçue. Selon Julien, cependant, une telle idée est une négation flagrante du libre arbitre humain ${ }^{47}$. Augustin répond succinctement, insistant sur le fait que Jean 1,12 montre que la potestas de devenir un enfant de Dieu est donnée par Dieu. Les êtres humains ne peuvent pas se l'accorder à eux-mêmes ${ }^{48}$.

\section{Conclusion}

Jean 1,12 parait être réparti proportionnellement dans la totalité de l'œuvre augustinienne. L'utilisation de ce verset pour établir un équilibre entre les pôles divin et humain de la foi se retrouve principalement dans ses premiers écrits (avant la controverse pélagienne), et surtout dans les Sermones 97A, 166, 360B. Néanmoins, bien que l'usage précoce de ce verset ne soit pas toujours clairement formulé et n'autorise pas toujours un lien spécifique avec la fides, on le trouve souvent en relation avec la grâce. Augustin insiste, en d'autres termes, sur le fait qu'être adopté comme enfant de Dieu est un effet de la grâce. L'Enarratio in Psalmum 120 (et dans une moindre mesure également l'Enarratio in Psalmum 72), au contraire, est une première occurrence de la formulation de la foi comme une grâce, sur la base de Jean 1,12. Cette exégèse est évidemment caractéristique de la plupart des écrits anti-pélagiens et des sermons situés dans la même période (les Sermones 143; 306; le Tractatus 53). Dieu conduit les personnes humaines au choix de la foi et les aide à vivre la vie d'un croyant.

46. Augustin, c. ep. Pel. 1.6. [CSEL 60:428/10-16].

47. Augustin, c. Iul. imp. 1.94.

48. Augustin, c. Iul. imp. 3.106 


\section{JEAN 6,44}

\section{Les écrits et les sermons antérieurs à la controverse pélagienne (avant 411/412)}

La grande majorité des références à Jean 6,44 se retrouvent dans les écrits anti-pélagiens et les écrits de l'époque de la controverse pélagienne. Seul un petit nombre de références précède la controverse, et certaines de ces références ne traitent pas de la fides ${ }^{49}$. À deux reprises - dans les sermons précédant la controverse - Jean 6,44 apparaît dans le contexte de la distinction entre les Juifs et les gentes où il est utilisé pour expliquer que la conversion des gentes est le travail de Dieu ${ }^{50}$. Dieu, comme le père de la parabole du fils prodigue (Luc 15,28), s'efforce de convaincre le fils aîné récalcitrant (symbolisant les Juifs) d'entrer, en utilisant les appels et les arguments (et non pas les injonctions) ${ }^{51}$.

\section{Les traités anti-pélagiens (après 411/412)}

Dans l'œuvre anti-pélagienne, le verset est utilisé afin de démontrer - parfois implicitement ${ }^{52}$, mais dans la plupart des cas explicitement - que la foi nous est donnée comme un don. Dans $D e$ gratia Christi et de peccato originali (418), Augustin observe que la foi, comme c'est évident à partir de Jean 6,44 , est la grâce même que, selon lui, Pélage devrait reconnaître. Il insiste, en d'autres termes, sur sa thèse selon laquelle la foi est un don de la grâce ${ }^{53}$. Le Contra duas epistulas Pelagianorum (421) est encore plus spécifique dans sa revendication d'une foi qui ne dépend pas d'un mérite humain. Il est faux d'argumenter que les gens sont aidés par l'acquisition de la potestas pour devenir les enfants de Dieu, ayant mérité ladite potestas en premier lieu en recevant le Christ sur la base de leur libre arbitre et sans l'aide de l'une ou l'autre grâce. Ceux qui émettent cette

49. Augustin, diu. qu. 38 (388-396); ep. 93.5 (407-408).

50. Augustin, en. Ps. 134.22 (Müller: -, Zarb: la seconde moitié de 411-412, La Bonnardière: 408-411, Hombert : 403-404).

51. Augustin, s. 112A.11 ( $s$. Caillau 2.11.11, Rebillard: près de 400, Gryson: 403-404, Hombert: 403-404).

52. Augustin, perf. iust. 41 (415). Les mots de Jean 6,44 sont prononcés par la source de la foi - le Christ - et renvoient à la foi avec laquelle nous croyons en lui. Cette affirmation implique que la foi est un don.

53. Augustin, gr. et pecc. or. 1.11 . 
affirmation soutiennent, de manière erronée, que la grâce est accordée en fonction du mérite. Jean 1,12 est également mal interprété en ce sens que la foi (antérieure et librement choisie) mérite la grâce ( $c f$. supra). Augustin emploie Jean 6,44 et Jean 6,64-65 comme des contre-arguments, en insistant sur le fait que la foi est aussi donnée ${ }^{54}$. Il ajoute dans le même document, de nouveau sur base de Jean 6,44, que le début de notre bonne volonté et la foi doivent être trouvés en Dieu et non pas en nous-mêmes ${ }^{55}$. Il défend une position similaire dans le De praedestinatione sanctorum (428-429). Ceux qui croient, entendent l'Évangile à la fois de l'extérieur et de l'intérieur, et ils reçoivent le don de la foi. «Être attiré par le Père» signifie recevoir du Père le don de la foi dans le Christ ${ }^{56}$. Dans la lettre 194 (418-419) à Sixte (le pape suivant), avec son accent anti-pélagien, Augustin insiste sur base de Jean 6,44 pour affirmer que la foi nous porte vers le Christ et que cette foi nous est accordée comme un don ${ }^{57}$.

À l'une ou l'autre occasion, Jean 6,44 apparaît dans le corpus anti-pélagien en dehors du contexte de la fides strictement dit, se

54. Augustin, c. ep. Pel. 1.6. Augustin écrit le texte suivant dans un contexte clairement régi par la conviction que la foi doit être considérée comme un don de Dieu: «Et hoc propter credentes et non credentes se dixisse manifestauit exponens quod dixerat: nisi Pater, qui misit me, traxerit eum [Jean 6,44], id ipsum aliis uerbis repetendo in eo, quod ait: nisi fuerit ei datum a Patre meo [Jean 6,66]. Ille quippe trahitur ad Christum, cui datur ut credat in Christum. Datur ergo potestas, ut filii Dei fiant qui credunt in eum, cum hoc ipsum datur, ut credant in eum. Quae potestas nisi detur a Deo, nulla esse potest ex libero arbitrio, quia nec liberum in bono erit, quod liberator non liberauerit, sed in malo liberum habet arbitrium, cui delectationem malitiae uel occultus uel manifestus deceptor inseuit uel sibi ipse persuasit» [CSEL 60:428/5-15].

55. Augustin, c. ep. Pel. 1.37. Augustin explique que le début de la volonté de faire le bien n'est pas à trouver dans la personne humaine. Si ceci était le cas, alors la grâce serait méritée. Il se réfère en ce cas à l'exemple de Saul. Où était chez Saul - le persécuteur des chrétiens - la bonne volonté qui méritait sa conversion? C'est pour cette raison que les croyants doivent prier - selon le commandement du Seigneur - pour ceux qui les persécutent. Nous ne devons pas prier pour qu'ils reçoivent la grâce de Dieu en échange de leur bonne volonté. Nous devons plutôt prier, pour que leur mauvaise volonté soit changée en bonne volonté.

56. Augustin, praed. sanct. 15. «Cum igitur euangelium praedicatur, quidam credunt, quidam non credunt: sed qui credunt Praedicatore forinsecus insonante, intus a Patre audiunt atque discunt; qui autem non credunt, foris audiunt, intus non audiunt neque discunt: hoc est, illis datur ut credant, illis non datur. Quia nemo, inquit, uenit ad me, nisi Pater qui misit me, traxerit eum [Jean 6,44]» [PL 44:972/19-25].

57. Augustin, ep. 194.12. «Fides igitur ad Christum nos trahit, quae nisi desuper gratuito munere nobis daretur, non ipse diceret: nemo potest uenire ad me, nisi Pater, qui misit me, traxerit eum [Jean 6:44]» [CSEL 57:185/16-18]. 
référant à la prédestination en général. Dans le Contra Iulianum (421-422), Augustin déclare en se fondant sur la péricope que seuls ceux que Dieu voudra sauver seront sauvés. En d'autres termes, seuls ceux qui sont attirés par Dieu sont sauvés ${ }^{58}$. Dans le même ordre d'idées, et aussi sans allusion explicite à la fides, Augustin fait remarquer dans l'Epistula 217 (426-428) que la grâce n'est pas (seulement) le don du libre choix, de la loi, de l'enseignement, comme d'après lui les pélagiens le présentent de manière erronée. La grâce est accordée par la volonté de Dieu à toute action humaine individuelle ${ }^{59}$.

\section{Les sermons contemporains ou liés à la controverse pélagienne (après 411/412)}

Les sermons citant Jean 6,44 sont le plus souvent datés de l'époque de la controverse pélagienne. L'Enarratio in Psalmum 87.10 (de $418)^{60}$ est encadrée dans le contexte de l'idée selon laquelle seule la grâce de Dieu peut apporter la vie à ceux qui sont spirituellement morts. Augustin cite Jean 6,44, à ce moment, concluant que la foi - en laquelle nous croyons et à travers laquelle l'âme est ramenée à la vie après la mort du cœur - nous est accordée par Dieu.

In Iohannis euangelium tractatus 26 (414) est situé à l'époque de la controverse pélagienne. Augustin utilise Jean 6,44 dans ce sermon pour décrire la foi comme un don de Dieu d'une part, et comme un acte libre de la personne humaine de l'autre. La foi est la coopération entre Dieu qui attire l'homme, et l'homme qui répond à cette attraction par son libre arbitre. Ceux qui ne sont pas «attirés», ne parviennent pas à la foi. En ce sens, parvenir à la foi est une grâce. Nous ne devrions pas essayer de comprendre pourquoi Dieu choisit d'attirer certaines personnes à lui et pas d'autres ${ }^{61}$. Augustin

58. Augustin, $c$. Iul. $4.44 ; 5.14$. Augustin ne considère pas que cette «élection» est une violation de la justice de Dieu. En fait, ce serait juste que tous devraient être punis, car nous sommes tous pécheurs (à l'exception du Christ). Le fait que pourtant Dieu nous sauve est une expression de sa miséricorde.

59. Augustin, ep. 217.12.

60. Müller, La Bonnardière, Zarb, Rondet.

61. Cette idée est similaire à l'utilisation par Augustin de Rom. 9,20; 11,33 versets qu'il ne cite pas, à ce stade - où il tente d'expliquer (en particulier durant la soi-disant controverse semi-pélagienne) qu'il ne comprend pas pourquoi Dieu en sauve certains et pas d'autres. 
recommande la prière à ceux qui n'ont pas encore été attirés vers $D^{2} u^{62}$. Cependant être attiré vers la foi et l'embrasser ne peut pas se faire sans la volonté de l'homme ${ }^{63}$ : on ne peut être attiré malgré soi (inuitus) ${ }^{64}$. Ce trahere est: «non necessitas, sed uoluptas, non obligatio, sed delectatio $[\ldots] \gg^{65}$. L'équilibre entre les dimensions divines et humaines de la foi est également évident quand Augustin déclare plus tard dans un sermon que ceux qui croient que le Fils est égal au Père seront attirés vers le Fils par le Père. L'âme aspire à la vérité et, ce faisant, elle est attirée vers la vérité ${ }^{66}$.

Dans le Sermo $30.10(417)^{67}$, Augustin juxtapose Jean 6,44 et 1 Cor. 4,7 («Quid enim habes, quod non accepisti?») comme une preuve évidente que Dieu nous a accordé tout ce que nous avons à travers l'amour, et il utilise les deux passages pour fournir un commentaire en deux étapes sur Matt. 11,28-29 («Venite ad me omnes qui laboratis et onerati estis, et ego uos reficiam, tollite iugum meum super uos» $)^{68}$. Il commence en déclarant que l'expression «venir au Seigneur» signifie la foi dans le Seigneur. Dans le contexte plus large de Jean 6,44, il insiste sur le fait que ce

62. Augustin, Io. eu. tr. 26.2.

63. Augustin, Io. eu. tr. 26.2-3. En $\S 3$ Augustin observe que nous croyons avec le cœur.

64. Augustin, Io. eu. tr. 26.3.

65. Augustin, Io. eu. tr. 26.4. «Inde et hic si aduertis: nemo uenit ad me, nisi quem Pater attraxerit [Jean 6,44]. Noli te cogitare inuitum trahi; trahitur animus et amore. Nec timere debemus ne ab hominibus qui uerba perpendunt, et a rebus maxime diuinis intellegendis longe remoti sunt, in hoc scripturarum sanctarum euangelico uerbo forsitan reprehendamur, et dicatur nobis: quomodo uoluntate credo, si trahor? Ego dico: parum est uoluntate, etiam uoluptate traheris. Quid est trahi uoluptate? Delectare in Domino, et dabit tibi petitiones cordis tui [Ps. 36,4]. Est quaedam uoluptas cordis, cui panis dulcis est ille caelestis. Porro si poetae dicere licuit: trahit sua quemque uoluptas, non necessitas, sed uoluptas; non obligatio, sed delectatio, quanto fortius nos dicere debemus trahi hominem ad Christum, qui delectatur ueritate, delectatur beatitudine, delectatur iustitia, delectatur sempiterna uita, quod totum Christus est?» [CChrSL 36:261/15-262/27].

66. Augustin, Io. eu. tr. 26.5. Augustin ajoute ici qu'Arius n'a pas été attiré (au Fils, à la vérité) parce qu'il ne voyait pas le Fils comme égal au Père. Il fait allusion à cet égard à Matt. 16,17, où le Christ dit à Pierre que son intuition - que Jésus était le Christ, le Fils de Dieu - lui a été révélé. Pierre a ainsi été attiré (adtractio) par l'intermédiaire de ladite révélation (reuelatio).

67. Rebillard: 412-416, Gryson: septembre/octobre 416, Hombert: septembreoctobre 416, Partoens : à la fin de septembre-début octobre 417. Gert Partoens (ed., introd.), Sancti Aurelii Augustini. Sermones in epistolas apostolicas. Sermones CLI-CLVI, [CChrSL 41Ba], (Turnhout, 2007), xix.

68. Augustin, s. 30.9. [CChrSL 41:388/203-205.] 
que les croyants atteignent dans cette vie est toujours le résultat de la foi. Dans la ligne de 1 Cor. 4,7, il exprime alors sa conviction que les personnes humaines ne peuvent pas venir à Dieu de leur propre chef. Ce fait de venir à Dieu, par conséquent, n'est pas notre propre œuvre. Augustin déclare explicitement que la iustitia n'est pas le résultat du libre arbitre humain et que tout est reçu de Dieu. En combinant l'idée que le uenire signifie credere et que ce uenire nous est donné, il est donc possible pour le public d'Augustin de conclure de son homélie que la foi vient du Seigneur, bien que le prédicateur ne le dise pas explicitement ${ }^{69}$.

Le Sermo 131 a été prononcé le dimanche 23 septembre 417 à Carthage, ${ }^{70}$ contre les inimici gratiae Christi, ${ }^{71}$ et affirme que la croyance est une grâce: «... ipsum credere doni esse, non meriti...». ${ }^{72}$ Augustin justifie cette position en utilisant Jean 6,65 («nemo uenit ad me, nisi cui datum fuerit a Patre meo») et Jean 6,44. Le Père ne conduit (ducere) pas des personnes humaines à Luimême, Il les attire plutôt (trahere). ${ }^{73}$ Cette uiolentia parle au cœur, pas à la chair, et elle est dulcis, suauis plutôt que dure ou douloureuse.

69. Augustin, s. 30.10. «Regit te sub iugo suo et sub sarcina sua. Vt enim sit tibi sarcina eius leuis et iugum eius suaue, ille tibi amorem inspirauit. Amanti suaue est; non amanti durum est. Amanti suaue est: Dominus dedit suauitatem [Ps. 84,13]. An forte quia uel uenisti audiens: Venite ad me [Matt. 11,28], hoc ipsum tibi arrogare intendis, quia uenisti? "Ecce" inquis "ueni ad illum arbitrio meo, uoluntate mea. Quia ueni, reficit me. Quia ueni, iugum suum imponit mihi suaue. Quia dat amorem, sarcinam suam imponit mihi leuem amanti et diligenti. Haec omnia fecit in me, sed quia ueni ad eum." Ergo hoc sapis, quia uenisti tu tibi hoc praestitisti? Quid enim habes, quod non accepisti [1 Cor. 4,7]? Quomodo uenisti ? Credendo uenisti, sed nondum peruenisti. Adhuc in uia sumus. Venimus, sed nondum peruenimus. Seruite Domino in timore, et exultate ei cum tremore, nequando irascatur Dominus, et pereatis de uia iusta [Ps. 2,11-12]. Time ne cum tibi arrogas quia inuenta est a te uia iusta, ipsa arrogantia pereas de uia iusta. "Ego" inquit "ueni, arbitratu meo ueni, uoluntate mea ueni”. Quid turgescis? Quid tumescis? Vis nosse quia et hoc praestitum est tibi? Ipsum audi uocantem: Nemo ad me uenit nisi Pater qui me misit traxerit eum [Jean 6,44]» [CChrSL 41:388/209-389/229].

70. Rebillard: 23/09/417, Gryson: 23/09/417, Hombert: -, Partoens: 23/09/417.

71. Augustin, s. 131.9

72. Augustin, s. 131.2. [PL 38:730/8].

73. «Après 396, Augustin juge qu'on vient à la foi parce qu'on est appelé (Simpl. 1.2.10). Alors que la croyance est volontaire (spir. et litt. 30.52-32.56), c'est basé sur l'appel ou la persuasion de l'Unique en qui on doit croire (34.60) ». Augustin argue de cela avec Jean 6,44. Ce n'est pas un schéma qui implique la necessitas/obligatio, mais cela arrive par uoluptas/delectatio, en suscitant un consentement volontaire (Augustin, Io. eu. tr. 26.3). Eugene TeSelle, «Faith», Augustine through the Ages (ed. Allan D. Fitzgerald), 347-350, 349. 
C'est cette suauitas qui nous attire. Augustin compare ainsi l'homme sauvé par la grâce à un mouton qui est attiré par la vue de l'herbe lorsqu'il a faim. Le mouton n'est pas physiquement («corpore») obligé d'aller au pré, il est plutôt attiré par un désir («desiderio») ${ }^{74}$. La foi est une responsabilité: Augustin appelle son public à croire dans le crucifié, pour résister à la tempête orageuse des épreuves de la vie et des tentations. Il demande à ses auditeurs de croire au Christ, car: «ubi credis, ibi uenis $»^{75}$. En même temps, cependant, la foi est la grâce, parce que la croix nous assure que nous ne coulons pas. Augustin souligne, par ailleurs, que ceux qui ne montent pas à bord du navire de la foi n'ont qu'eux-mêmes à blâmer. Néanmoins, ceux qui montent à bord ne devraient pas se l'attribuer à eux-mêmes (arrogere). Augustin fait allusion ici à Jean 6,66. En d'autres termes, il insiste sur l'idée que la foi est un don et que cette compréhension devrait nous inciter à l'humilité et à la gratitude ${ }^{76}$. Dans le reste du sermon, Augustin attire l'attention sur le fait que nous avons reçu la iustitia de Dieu comme gratia et que Dieu œuvre en nous.

\section{Conclusion}

L'allusion à Jean 6,44 est limitée presque exclusivement au cadre anti-pélagien. C'est la référence biblique qu'Augustin emploie le plus explicitement et qu'il élabore plus systématiquement afin de démontrer que la foi n'est pas une activité humaine. La gratia divine est à l'initiative de la foi humaine. En ce sens, les Sermones 30 et 131, 1'In Iohannis euangelium tractatus 26 et l'Enarratio in Psalmum 87 sont en complet accord avec les traités anti-pélagiens.

\section{UN TRAITEMENT UNIQUE HOMILÉTIQUE DE LA GRATIA FIDEI}

Globalement, nous avons observé concernant Jean 1,12 qu'Augustin utilise déjà dans ses premiers écrits le verset comme

74. Augustin, s. 131.2. «Ista uiolentia cordi fit, non carni. Quid ergo miraris? Crede, et uenis; ama, et traheris. Ne arbitreris istam asperam molestamque uiolentiam: dulcis est, suauis est; ipsa suauitas te trahit. Nonne ouis trahitur, cum esurienti herba monstratur? Et puto quia non corpore impellitur, sed desiderio colligatur». [PL:38 730/19-20].

75. Augustin, s. 131.2. [PL 38:730/20.]

76. Augustin, s. 131.3 . 
un topos appliqué à la grâce. Dans notre analyse de la «première» utilisation de ce verset, nous avons aussi noté, cependant, qu'Augustin met quelquefois l'accent sur l'expression «credentibus in eum», en utilisant le verset principalement pour indiquer qu'il relève de notre responsabilité de tenir une fides correcte, afin que Dieu puisse nous transformer. Ce faisant, toutefois, Augustin peut prêter moins d'attention au caractère de don de la foi, mais il ne le nie pas. L'accent mis sur la responsabilité humaine disparaît avec le début de la controverse pélagienne, après quoi l'attention se concentre presque exclusivement sur le segment précédent du verset, «dedit illis potestam filios Dei fieri». Ceci s'avère particulièrement le cas dans les sermons anti-pélagiens et les écrits contre Julien et le «semi-pélagianisme». L'interprétation de la grâce dans le verset se retrouve également dans les traités ultérieurs qui ne présentent pas une tendance anti-pélagienne. En tenant compte de toutes les nuances nécessaires, nous pouvons donc affirmer que Jean 1,12 fait toujours allusion à la grâce, pour Augustin, et que cela devient encore plus emphatique après 412 quand cette allusion est appliquée à la fides.

La moindre présence, voire la non-thématisation du sujet de la fides comme grâce dans les sermons d'Augustin, observées dans le contexte de son exégèse de Jean 1,12, n'impliquent pas qu'il n'a pas considéré cela comme important ou que ses sermons ont maintenu une opinion différente de ses traités sur cette question. Comment pouvons-nous alors expliquer que le thème de la gratia fidei soit moins présent? Une première hypothèse est que peu ou pas de sermones ad populum n'ont subsisté dans lequel le thème de la foi de la controverse pélagienne est traité. Une autre explication suggère qu'Augustin se limitait probablement à un dialogue écrit sur le sujet des pélagiens et ne le répétait pas dans sa prédication. Il est en effet possible qu'Augustin n'ait pas voulu troubler les fidèles avec la question, ou qu'il ait voulu éviter les agitations théologiques. Dans le même ordre d'idée, on peut se demander si le silence d'Augustin sur le caractère de don de la fides dans les sermons qui traitent de la foi mais concentrent l'attention sur la dimension humaine de ce don présente une quelconque signification. Il va sans dire que l'absence de référence explicite à l'initiative divine dans les sermons d'Augustin ne doit pas nous amener à conclure qu'il ne considère pas l'acte humain de la foi comme étant enraciné dans 
cette grâce. Par ailleurs, la référence occasionnelle à la gratia fidei dans ses sermons - comme nous l'avons observé en particulier dans le cas de Jean 6,44 - démontre qu'Augustin n'avait pas élaboré une doctrine différente de la grâce dans le contexte homilétique. Il n'établit pas une distinction «tactique» entre ses écrits traitant du caractère exhaustif de la grâce et ses sermons, où il se concentre sur l'initiative humaine pour des raisons pastorales. Il est possible, néanmoins, que ces considérations pastorales et la différence de genre entre les écrits descriptifs et les homélies exhortatives puissent offrir une hypothèse explicative complémentaire à propos du traitement différencié de la fides, à savoir que le public d'Augustin était composé de croyants et qu'il ne considérait pas toujours nécessaire d'entrer dans les détails sur la source de la foi humaine; ses sermons acceptent implicitement la foi comme un don. D'un point de vue rhétorique, par conséquent, il ne voyait aucune raison d'explorer le sujet avec des gens qui croyaient déjà, mais il préférait attirer l'attention sur la signification de la foi active en tant que responsabilité humaine.

Jean 6,44 est clairement un topos anti-pélagien, car il ne semble pas apparaître d'une manière significative avant la controverse. Il est intéressant d'observer que la désignation de la foi en tant que grâce (la conséquence du trahere de Dieu) sur base de ce verset se déroule en premier lieu dans le genre homilétique. Une élaboration initiale sans équivoque de ce thème se trouve dans l'In Iohannis euangelium tractatus 26 (414), une localisation assez évidente si l'on garde à l'esprit qu'Augustin a dédié cette série de sermons à l'explication de l'Évangile de Jean. Néanmoins, il représente déjà une explication systématique du sens du trahere divin, qu'Augustin théorisera explicitement dans les Sermones 30 et 131 (les deux en 417) et par la suite dans le De gratia Christi (418). Le traitement de Jean 6,44 par Augustin indiquait la continuité entre le genre des sermons et celui des traités systématiques/polémiques de la gratia. La gratia fidei est traitée dans les deux genres de la même manière, dans le cadre de son exégèse du verset.

Notre analyse de l'utilisation de Jean 1,12 et 6,44 par Augustin vient ainsi à l'appui des études de Hombert et d'Ogliari. L'étude de Jean 1,12 a illustré le fait qu'Augustin avait examiné la question de la grâce bien avant 411/412, mais que l'accent spécifique mis sur la foi comme grâce liée à cette péricope survient seulement 
après le début de la controverse pélagienne. Le fait que la présence de Jean 6,44 dans l'œuvre d'Augustin, verset qui est cité très souvent pour souligner la gratia fidei, est principalement limitée aux sermons/écrits antérieurs à 411/412 confirme cette thèse. Nous avons cependant remarqué une exception frappante. L'Enarratio in Psalmum 120 (405-411) explique en association avec Jean 1,12 que la potestas de la foi et de sa protection - la persévérance - est quelque chose que nous recevons. Formulé différemment, il s'agit d'une apparition précoce de la pensée d'Augustin selon laquelle l'initium fidei et la perseuerantia fidei sont deux expressions de la grâce de Dieu. L'exégèse augustinienne de Jean 1,12 après 411/412 et de Jean 6,44 (qui est presque limitée à cette période) confirme les conclusions d'Ogliari, à savoir que les sermons traitent la grâce - et dans ce cas spécifique, la gratia fidei - au même niveau spéculatif que dans le traité anti-pélagien et avec le même accent porté sur ladite gratia.

Au terme de notre analyse, nous avons démontré qu'il existe une certaine continuité dans la pensée d'Augustin sur la grâce. La question était déjà présente bien avant le début de la controverse pélagienne. Il s'agit toutefois d'une continuité en développement. Son premier traitement de la grâce de la foi ne négligeait pas le pôle divin, mais s'intéressait plutôt au pôle humain, tandis que plus tard l'accent se déplace, principalement du fait des questions posées dans la controverse pélagienne. Deuxièmement, nous avons observé que les sermons situés à cette période sont liés, en termes de contenu et dans leur utilisation de l'Écriture, aux écrits antipélagiens. Nous avons remarqué la continuité entre les genres. Dans le genre pastoral, nous avons trouvé le même contenu de la grâce que dans la systématique, bien que la perspective soit parfois adaptée au genre. Les sermons ne dénient pas la foi comme étant donnée par Dieu, mais consacrent cependant une attention plus grande à la responsabilité humaine, alors que les traités font parfois l'inverse. Il est ainsi devenu évident que certains sermons préfèrent une exégèse alternative des mêmes citations employées par les traités. Cette distinction sert en première instance à illustrer l'originalité littéraire du sermon, qui prend l'Écriture comme point de départ inspirant et structurant un discours rhétorique construit dans un contexte liturgique avec l'intention explicite d'exhorter les fidèles à une pratique chrétienne et à la foi. En outre, les mêmes 
thèmes relatifs au contenu sont parfois traités différemment, en fonction du but rhétorique et de l'objectif pastoral propre au genre du sermon. En bref, il est devenu évident que les sermons sont liés aux écrits systématiques et, qu'en même temps, ils traitent de la gratia fidei d'une manière qui leur est propre et unique ${ }^{77}$.

Anthony.Dupont@theo.kuleven.be

KULeuven - Faculty of Theology, Research Unit History of Church and Theology

Sint-Michielsstraat 4 bus 3101, B-3000 Leuven, Belgium

77. J'adresse mes remerciements à M. Pierre Descotes (Université Paris IV Sorbonne) pour ses précieuses remarques de lecture. 